\title{
Thiazolidinedione Use and Ulcerative Colitis-related Flares: An exploratory analysis of administrative data
}

\author{
Jennifer L. Lund, MSPH ${ }^{\star}$, Til Stürmer, MD, MPH ${ }^{*}$, Carol Q. Porter, BS ${ }^{\dagger}$, Robert S. Sandler, \\ MD, $\mathbf{M P H}^{*}, \ddagger$, and Michael D. Kappelman, MD, MPH ${ }^{\S}$ \\ * Department of Epidemiology, University of North Carolina, Gillings School of Global Public \\ Health, Chapel Hill, North Carolina \\ † Cecil G. Sheps Center for Health Services Research, University of North Carolina, Chapel Hill, \\ North Carolina \\ ‡ Department of Medicine, Division of Gastroenterology and Hepatology, University of North \\ Carolina, School of Medicine, Chapel Hill, North Carolina \\ $\S$ Division of Pediatric Gastroenterology, Department of Pediatrics, University of North Carolina, \\ School of Medicine, Chapel Hill, North Carolina
}

\begin{abstract}
Background-Recent animal studies and clinical trials suggest that thiazolidinediones, a class of oral antidiabetic agents, are efficacious in reducing inflammation, yet no studies have evaluated their effectiveness in preventing flares. We examined the association between thiazolidinedione use and ulcerative colitis (UC)-related flares.

Methods-We conducted a retrospective cohort study using administrative data from 87 health plans across 33 states. Individuals with both UC and diabetes were identified using administrative definitions. Exposure to thiazolidinediones or other oral antidiabetic agents was ascertained through outpatient pharmacy claims. The primary outcome was occurrence of a UC flare defined by: 1) a new prescription for oral steroids, infliximab, or oral/rectal salicylates, or 2) a claim for colectomy. Secondary analyses analyzed outcomes separately. We estimated hazard ratios (HRs) and $95 \%$ confidence intervals (CIs) using Cox proportional hazards regression after matching each thiazolidinedione user to a comparable oral antidiabetic user on propensity score.
\end{abstract}

Results-This study included 142 thiazolidinedione and 468 other oral antidiabetic users with a mean follow-up of 7.3 and 6.2 months, respectively. Thiazolidinedione use was not associated with UC-related flares as measured by the composite outcome (HR $=1.05,95 \%$ CI: $0.66,1.68)$. However, thiazolidinedione use was associated with a nonsignificant reduction in risk of oral steroid use when analyzed as a separate outcome ( $\mathrm{HR}=0.53,95 \% \mathrm{CI}: 0.20,1.44)$.

Conclusions-Thiazolidinediones do not provide any benefit over other oral antidiabetics in preventing UC-related flares as measured by our primary composite outcome. However, thiazolidinedione use may reduce the risk of more significant disease flares requiring oral steroid treatment.

Copyright () 2010 Crohn's \& Colitis Foundation of America, Inc.

Reprints: Jennifer L. Lund, MSPH, Department of Epidemiology, Gillings School of Global Public Health, University of North Carolina at Chapel Hill, McGavran-Greenberg Hall, CB\# 7435, Chapel Hill, NC 27599-7435 (jlund@email.unc.edu).

Additional supporting information may be found in the online version of this article. 


\section{Keywords}

ulcerative colitis; thiazolidinediones; epidemiology

Ulcerative colitis (UC) is a chronic inflammatory bowel disease (IBD) that affects nearly 500,000 Americans. ${ }^{1,2}$ The clinical course is typically relapsing and remitting: patients experience flares of their illness with symptoms of abdominal pain, diarrhea, rectal bleeding, and extra-intestinal manifestations, followed by periods of remission. Thus, the goals of therapy are twofold: 1) to treat disease flares (induction of remission) and 2) to prolong the length of time between flares (maintenance of remission). Despite the morbidity and mortality associated with $\mathrm{UC},{ }^{3}$ limited treatment options exist and additional therapeutic agents are needed. Thiazolidinediones, inhibitors of PPAR gamma, ${ }^{4}$ were introduced to the US market in 1997. Although currently indicated for the treatment of type 2 diabetes mellitus ("diabetes"), 5 preclinical data and a recent randomized controlled trial have demonstrated the efficacy of rosiglitazone (one member of the thiazolidinedione class) for the induction of remission in UC patients with active disease. ${ }^{6-9}$ Yet the effectiveness of thiazolidinediones in the maintenance of $\mathrm{UC}$ remission has not been comprehensively evaluated.

Diabetes is one of the most common chronic illnesses in the US, with a prevalence of $8 \%{ }^{10}$ Based on the combined prevalence of UC and diabetes, a substantial percentage of Americans may be affected by both conditions. UC may be particularly problematic in patients with coexisting diabetes because oral steroids, a mainstay of UC treatment, can exacerbate hyperglycemia. Therefore, preventing UC flares in these patients is of particular importance.

Thiazolidinediones are considered second-line oral medications, reserved for diabetics who fail to achieve metabolic goals on metformin therapy. However, if thiazolidinediones are effective in maintaining UC remission in diabetic patients, there would be a strong argument to move this class to first-line therapy in diabetic patients with UC. Additionally, since few medications have been shown to maintain remission in UC, thiazolidinediones could have a role in the primary treatment of UC patients with or without diabetes. Therefore, we performed an exploratory retrospective cohort study using administrative data to examine the association between thiazolidinedione use and UC flares in patients with UC and diabetes.

\section{MATERIALS AND METHODS}

\section{Data Source}

We conducted a retrospective cohort study using administrative claims data from the PharMetrics Patient-Centric Database (IMS Health, Watertown, MA) during January 1, 2000 to December 31, 2005. This longitudinal, individual-level database included 87 health plans across 33 states in the US. Data included inpatient and outpatient diagnoses and procedures, as well as retail and mail-order prescription records. Demographic information and start and stop dates for plan enrollment and pharmacy benefits were available. All records used in the analysis were deidentified; therefore, this study was exempt from UNC Institutional Review Board review.

\section{Sample Population}

Individuals in the study cohort were required to 1) be 18 to 64 years old, 2) meet the administrative definitions for both diabetes and UC during the time period from January 1, 1995 to May 31, 2005, and 3) have at least 180 days of claims history prior to study entry. 
We defined diabetic individuals as having two or more claims of ICD-9 250.XX and at least one oral antidiabetic medication dispensed. ${ }^{11} \mathrm{UC}$ was defined by having three or more UC claims (ICD-9 556.XX), or one IBD medication and one UC claim. ${ }^{2}$

\section{Exposure and Outcome Definitions}

Our primary analysis included a cohort of prevalent users of the following oral antidiabetic medication classes: thiazolidinediones (rosiglitazone and pioglitazone), meglitinides, nateglinides, sulfonylureas, biguanides, exenatides, and alpha-glucose inhibitors. Individuals were classified as "thiazolidinedione users" if they were dispensed either rosiglitazone or pioglitazone alone or in combination with another oral antidiabetic drug, otherwise, they were classified as "other oral antidiabetic users."

In a secondary analysis we restricted the study population to a subcohort of "new-users" of oral antidiabetic agents (i.e., individuals without a prescription for an oral antidiabetic medication dispensed in the 90 days prior to study entry). ${ }^{12}$

Due to the lack of clinical information in administrative databases, the primary study outcome, the occurrence of a UC-related flare, was defined as any of the following events: 1) a new prescription dispensed for an oral steroid (days supplied $\geq 14$ ), infliximab, or an oral or rectal salicylate, or 2) a claim for colectomy. For oral steroids, we required a minimum of 14 days supplied to increase the specificity of this definition for UC flares by excluding steroid use for other acute indications such as respiratory, dermatologic, or other inflammatory conditions. Individuals using oral or rectal salicylates during the 90 days prior to study entry (presumably those on maintenance therapy) were not eligible for the specific outcome of a new prescription for an oral/rectal salicylate. Subanalyses examined the outcomes of a new prescription for oral steroids and oral/rectal salicylates separately.

\section{Covariates}

Data on potential confounders were obtained from the inpatient, outpatient, and pharmacy claims and included age at study entry, sex, geographic region (East, Midwest, South, West), insurance type (Medicaid versus commercial), concomitant medication use (insulin, 6mercaptopurine, imuran, methotrexate, entocort, oral and rectal salicylates, and adalimumab), comorbidities (based on ICD-9 codes from the Deyo implementation of the Charlson comorbidity score, used separately, not as a score), calendar year, and healthcare utilization (total prescriptions in 180 days prior study entry [0-13, 14-27, >28] and number of days-in-claims 180 days prior to study entry $[0-5,6-11,>12])$.

\section{Cohort Entry and Exit}

Follow-up began on the day of the first oral antidiabetic prescription dispensed after the individual had one claim for UC, one claim for diabetes, and 180 days of claims history. Individuals who did not fulfill the administrative criteria for $\mathrm{UC}$ and diabetes were subsequently excluded from analysis. Individuals who had a prescription for an oral steroid or infliximab in the 90 days prior to study entry or a claim for colectomy in the 180 days prior to study entry were excluded. Follow-up continued until the occurrence of 1) the primary outcome, 2) a lapse in plan enrollment/pharmacy benefit of $>30$ days, 3) stopping of baseline oral antidiabetic medication, 4) end of the study period (May 31, 2005), or 5) reaching 65 years of age. Individuals who stopped taking their medication exited the cohort 30 days after the last dispensing (+ days supplied), which is usually 90 days after the last dispensing. Additionally, members of the other oral antidiabetic group exited the cohort if they started thiazolidinedione treatment (exit date was defined as the date that the thiazolidinedione prescription was dispensed). 


\section{Statistical Analyses}

Propensity Score Model Analysis-Descriptive statistics were reported for continuous variables as means with standard deviations and for categorical variables as counts and percentages. To control for measured confounders, we developed a propensity score model using the administrative claims data to quantify the probability that a person was treated with a thiazolidinedione. ${ }^{13}$ Covariates included in the propensity score were selected according to a two-step process. We first constructed an outcome model identifying independent predictors of UC-related flares $(P<0.1)$ using the variables shown in Table 1. Subsequently, we consulted subject matter experts to identify additional predictors of UCrelated flares. ${ }^{14}$ We used multivariate logistic regression to estimate propensity scores for each individual in the prevalent cohort and new-user subcohort separately. Using the predicted propensity scores from our model, we attempted to match all thiazolidinedione users with other oral antidiabetic users through 5-to-1 greedy matching. ${ }^{15}$ Matching on propensity score is one technique commonly used to control for measured confounding in observational studies.

Analysis-Analyses were performed for the prevalent cohort and the new-user subcohort separately. We estimated the association between thiazolidinedione use and UC-related flares using Cox proportional hazards regression before and after matching on propensity score. Subanalyses of the new oral steroid and oral or rectal salicylate outcomes were examined separately among prevalent users. All data analysis was performed in SAS 9.2 (SAS Institute, Cary, NC).

\section{RESULTS}

\section{Descriptive Statistics}

Using the administrative definitions described above, we identified 31,184 individuals with UC from January 1, 1995 to May 31, 2005. Within this group we identified 610 individuals from January 1, 2000 to May 31, 2005 with UC and diabetes, of which 142 were prevalent thiazolidinedione users and 468 were other oral antidiabetic users. Thiazolidinedione and other oral antidiabetic users had similar baseline characteristics, likely because oral antidiabetic prescribing is not influenced by factors associated with the UC-related flares. Comparing thiazolidinedione users to other oral antidiabetic users in the prevalent user cohort before propensity score matching, the mean age was 54 (SD 7.0) versus 53 years (SD 7.6), the percentage of females was $43 \%$ versus $44 \%$, and the regional distribution was East ( $25 \%$ versus $22 \%$ ), Midwest (30\% versus 34\%), South (32\% versus $24 \%$ ), and West (13\% versus $20 \%$ ). There were slightly more thiazolidinedione users with previously insulin use (19.7\% versus $9.2 \%$ ) and cardiovascular comorbidities (4.2\% versus $1.5 \%)$. The median duration of follow-up among prevalent thiazolidinedione users was 7.3 months, compared to 6.2 months for other oral antidiabetic users.

The new-user subcohort consisted of 260 individuals: 54 thiazolidinedione and 206 other oral antidiabetic users. Similar to the prevalent user cohort, baseline characteristics in the new-user subcohort did not substantially differ by medication group, with the exception of prior insulin use (18.5\% versus $4.4 \%)$ and comorbid diabetes with chronic complications (11.1\% versus $2.4 \%$ ) (data not shown). The median duration of follow-up in the new-user subcohort was 6.2 months among thiazolidinedione users and 6.4 months among other oral antidiabetic users.

\section{Propensity Score Model and Matching Results}

As a result of the first step in the covariate selection process, prior insulin and immunomodulator use and prior cardiovascular and peripheral vascular comorbidities were 
entered into the propensity score model. Next we added age at study entry, sex, region, number of days-in-claims, number of prescriptions in the 180 days prior to study entry, and calendar year at study entry to the final propensity score model based on expert opinion of clinically relevant factors. In the prevalent-user cohort, propensity scores ranged from 0.09 0.79 with a median of 0.24 for thiazolidinedione users and from $0.06-0.60$ with a median of 0.21 for other oral antidiabetic users. Using the 5-to- 1 matching algorithm, ${ }^{15}$ we matched 138 out of 142 (97\%) thiazolidinedione users to comparable other oral antidiabetic users (Supplementary Fig. 1, top panel).

In the new-user subcohort, propensity scores ranged from $0.06-0.83$ with a median of 0.28 for thiazolidinedione users and from $0.01-0.75$ with a median of 0.15 for other oral antidiabetic users. Within this cohort, we matched 50 out of 54 (93\%) thiazolidinedione users to comparable other oral antidiabetic users. (Supplementary Fig. 1, bottom panel).

Table 1 shows the distribution of baseline characteristics among the prevalent users in both exposure groups, after matching on propensity scores. As expected, the prevalences of the factors included in the propensity score model were well balanced across exposure groups. In addition, most other covariates were also balanced between the two exposure groups, with the exception of prior entocort use ( $0 \%$ versus $3.6 \%)$ and prior diagnosis of mild to moderate diabetes $(87.7 \%$ versus $76.1 \%)$. Similar results were found in the matched newuser subcohort (data not shown).

\section{Regression Model Results}

Table 2 reports the number of events, total person-years, and incidence rates of UC-related flares per 100 person-years by exposure group. These results are presented for the composite, new oral steroid, and new oral/rectal salicylate endpoints in the prevalent cohort and the new-user subcohort for the unmatched analysis. Multiple endpoints were reached on the same day. Among prevalent users, three had simultaneous oral steroid and oral salicylate prescriptions, two had oral steroid and rectal salicylate prescriptions, and two had oral and rectal salicylate prescriptions. Among new users, one had simultaneous oral steroid and rectal salicylate prescriptions and another had oral and rectal salicylate prescriptions.

Hazard ratios (HRs) and 95\% confidence intervals (CIs) for each endpoint comparing thiazolidinedione users to other oral antidiabetic users are reported in the two cohorts. In the crude analysis, the hazard for UC-related flares among individuals dispensed thiazolidinediones was similar to that among individuals dispensed other oral anti-diabetic medications in prevalent users ( $\mathrm{HR}=0.90,95 \% \mathrm{CI}: 0.63,1.30)$ and in new users $(\mathrm{HR}=$ $1.12,95 \% \mathrm{CI}: 0.64,1.95)$. After matching on propensity score, the hazard ratio remained relatively unchanged for prevalent users $(\mathrm{HR}=1.05,95 \% \mathrm{CI}$ : 0.66, 1.68) (Fig. 1A) and new users $(\mathrm{HR}=1.41,0.65,3.05)$.

We conducted subanalyses for the new oral steroid endpoint and new oral/rectal salicylate endpoint in the prevalent user cohort utilizing the original propensity score model, matching algorithm, and Cox proportional hazards model (Table 2). We were unable to replicate the subanalysis in the new-user subcohort due to small numbers. In the oral steroid outcome analysis we found a protective, non-significant association between thiazolidinedione use and a new oral steroid prescription when compared to other oral antidiabetic use before (HR $=0.46,95 \%$ CI: $0.20,1.08)$ and after $(\mathrm{HR}=0.53,95 \%$ CI; 0.20, 1.44) matching (Fig. 1B). A subanalysis of the outcomes of oral steroids and/or infliximab yielded essentially identical results (data not shown). In the oral/rectal salicylate analysis, we found no association between thiazolidinedione use and a new oral or rectal salicylate prescription before (HR $=$ $1.06,95 \%$ CI: $0.70,1.60)$ or after $(\mathrm{HR}=1.27,95 \%$ CI: $0.73,2.20)$ matching. 


\section{DISCUSSION}

We conducted a retrospective cohort study of individuals with UC and diabetes using a large administrative claims database to examine the effectiveness of thiazolidinediones, as compared to other oral antidiabetic medications, in preventing UC-related flares. In this exploratory analysis we found that the hazards for UC flares, indicated by a composite outcome including 1) a new prescription for oral steroids, infliximab, or oral/rectal salicylates, or 2) a claim for colectomy, were not different for individuals dispensed thiazolidinediones and other oral antidiabetic medications. However, a subanalysis of the outcome of oral steroids among prevalent users demonstrated a non-significant protective effect of thiazolidinediones. Although this study was underpowered to detect an effect in this subanalysis, the magnitude of this effect (adjusted HR $=0.53$ ) is quite substantial. Furthermore, the endpoint of oral steroid use is clinically meaningful (indicator of moderate to severe flares). We believe the findings of this exploratory study justify further examination of the possible protective effects of thiazolidinediones in larger cohort studies and/or clinical trials. However, thoughtful consideration of the overall risk/benefit trade-off is warranted in light of recent studies detailing an increased risk of cardiovascular and fracture risks among thiazolidinedione users. ${ }^{16-18}$

To implement our study we created a prevalent user cohort and new-user subcohort. The prevalent user cohort included individuals who may have been using oral antidiabetic drugs for a period of time prior to study entry. Including these individuals in our analysis increased the sample size and statistical efficiency, but may have introduced bias if the risk of the outcome varied with time on treatment. ${ }^{12,19}$ In addition, measured covariates prior to study entry may be affected by oral antidiabetic medication use, resulting in bias. ${ }^{12,20}$ To avoid these potential biases, we also conducted an analysis within a cohort of new users, individuals who did not use oral antidiabetic medications in the 90 days prior to study entry. This analysis confirmed the results of the prevalent user analysis. We also sought to minimize confounding by indication and "healthy user" bias by comparing thiazolidinedione users to other oral antidiabetic users rather than individuals not using diabetic medications. ${ }^{21}$ Lastly, we used propensity score matching based on a 5-to-1 greedy algorithm to maximize the number and quality of thiazolidinedione to other oral antidiabetic user matches. ${ }^{15}$ In both the prevalent user cohort and new-user subcohort, matches were made for over $90 \%$ of all thiazolidinedione users. The resulting HRs from the Cox regression models represent the effect of thiazolidinediones in thiazolidinedione users (or the "treatment effect in the treated"). ${ }^{22}$

Our study has several limitations that should be considered when interpreting the results. Our study population of individuals with UC and diabetes was defined using administrative, not clinical data, and is therefore subject to misclassification error. However, to minimize this bias we applied stringent algorithms for both conditions modeled after previously published criteria. ${ }^{1,2,12}$ The use of stringent inclusion criteria requiring multiple healthcare contacts and/or prescription medication use may limit the generalizability of these findings to patients with milder disease requiring less frequent medical care. However, exclusion of such individuals with milder disease should be nondifferential with respect to exposure status, and not introduce significant selection bias.

Our primary outcome measure was a composite marker for a UC-related flare. We made our best effort to identify pharmacy and procedure claims that reflected true clinical UC flares, but this measure is also subject to misclassification. Our definition was consistent with one utilized by Lewis et al in the General Practice Research Database, which defined an exacerbation of IBD to be a new prescription for either an oral aminosalicylate or an oral steroid. Restricting to new oral steroid prescriptions, the authors found an $85 \%$ positive 
predictive value for the administrative definition of a flare and clinical confirmation from a physician. ${ }^{23,24}$ Confounding by indication, based on severity of diabetes, is another possible limitation of this analysis. We compared users of thiazolidinediones with users of other oral antidiabetic medications, and every effort was taken to further minimize this possibility by analyzing several indicators of diabetes severity (prior insulin use, diabetes complications, and comorbid conditions based on the Deyo implementation of the Charlson Comorbidity Index). In addition, we could not control for unmeasured variables not contained within administrative data including lifestyle factors (diet, smoking, exercise) and family history of IBD. However, we have no reason to suspect that these factors would be associated with thiazolidinedione exposure and thus represent significant unmeasured confounding.

In this analysis we only studied the effectiveness of thiazolidinediones in UC patients with diabetes in a commercially insured population. Therefore, these results may not be generalizable to UC patients without comorbid diabetes or to noncommercially insured populations. Further, due to the relatively small number of thiazolidinedione users in the study and frequent switching between rosiglitazone and pioglitazone use, we were unable to compare the effectiveness of these two medications separately.

Despite these limitations, our study has a number of strengths. We were able to identify a diverse cohort of individuals with UC and diabetes encompassing a range of ages, regions, and comorbid conditions. Although our study did not randomly assign thiazolidinedione treatment, we were able to observe the potential effectiveness of the drug for preventing UC flares under "real-world" conditions. The use of propensity score matching allowed us to efficiently control for measured confounding, despite the relatively small number of outcomes in this study population.

All medication exposure information was determined based on outpatient pharmacy dispensing records, thus minimizing reliance on patient recall and/or physician documentation. Despite this advantage, we did not have information on patient compliance of the dispensed drug and were unable to capture medication use obtained through physician samples.

Finally, follow-up for our study was relatively long, averaging greater than 6 months in both exposure groups. A randomized controlled trial of this size would have been logistically and financially challenging to implement.

In conclusion, the results of this exploratory study suggest that thiazolidinedione use does not decrease the risk of total UC-related flares (defined by a composite outcome of new prescriptions for oral steroids, infliximab, oral/rectal salicylates, or colectomy), as compared to other oral antidiabetic use within a population of individuals with UC and diabetes.

However, we observed a nonsignificant tendency toward decreased oral steroid prescriptions in thiazolidinedione users, a marker for moderate to severe UC flares. Because few treatments for $\mathrm{UC}$ are currently available, pharmacoepidemiology studies such as this which evaluate the effectiveness of nongastrointestinal medications on the course of UC are especially valuable. These findings support the need for larger cohort studies and/or a prospective clinical trial to investigate the efficacy of thiazolidinediones in the maintenance of remission for patients with UC.

\section{Supplementary Material}

Refer to Web version on PubMed Central for supplementary material. 


\section{Acknowledgments}

Grant support: National Institutes of Health (grant T32 DK 07634 to J.L.L); grants KL2 RR025746 (to M.D.K.), P30 DK034987 (to R.S.S.), the UNC-GSK Center of Excellence in Pharmacoepidemiology and Public Health (to T.S.); unrestricted research grants from pharmaceutical companies to UNC (to T.S.); Robert Sandler serves as a consultant to Pozen, Inc.; Michael Kappelman receives research support from Centecor, Inc.

\section{References}

1. Herrinton LJ, Liu L, Lafata JE, et al. Estimation of the period prevalence of inflammatory bowel disease among nine health plans using computerized diagnoses and outpatient pharmacy dispensings. Inflamm Bowel Dis. 2007; 13:451-461. [PubMed: 17219403]

2. Kappelman MD, Rifas-Shiman SL, Kleinman K, et al. The prevalence and geographic distribution of Crohn's disease and ulcerative colitis in the United States. Clin Gastroenterol Hepatol. 2007; 5:1424-1429. [PubMed: 17904915]

3. The Crohn's and Colitis Foundation of America. www,ccfa.org

4. Fajas L, Auboeuf D, Raspe E, et al. The organization, promoter analysis, and expression of the human PPARgamma gene. J Biol Chem. 1997; 272:18779-18789. [PubMed: 9228052]

5. Food and Drug Administration. CDER. Orange Book: Approved Drug Products with Therapeutic Equivalence Evaluations. http://www.ac-cessdata.fda.gov/scripts/cder/ob/default.cfm

6. Dubuquoy L, Rousseaux C, Thuru X, et al. PPARgamma as a new therapeutic target in inflammatory bowel diseases. Gut. 2006; 55:1341-1349. [PubMed: 16905700]

7. Lewis JD, Lichtenstein GR, Deren JJ, et al. Rosiglitazone for active ulcerative colitis: a randomized placebo-controlled trial. Gastroenterology. 2008; 134:688-695. [PubMed: 18325386]

8. Liang HL, Ouyang Q. A clinical trial of combined use of rosiglitazone and 5-aminosalicylate for ulcerative colitis. World J Gastroenterol. 2008; 14:114-119. [PubMed: 18176972]

9. Su CG, Wen X, Bailey ST, et al. A novel therapy for colitis utilizing PPAR-gamma ligands to inhibit the epithelial inflammatory response. J Clin Invest. 1999; 104:383-389. [PubMed: 10449430]

10. Centers for Disease Prevention and Control: National Diabetes Surveillance System. National Diabetes Fact Sheet. 2007.

11. Rajagopalan R, Iyer S, Perez A. Comparison of pioglitazone with other antidiabetic drugs for associated incidence of liver failure: no evidence of increased risk of liver failure with pioglitazone. Diabetes Obes Metab. 2005; 7:161-169. [PubMed: 15715889]

12. Ray WA. Evaluating medication effects outside of clinical trials: new-user designs. Am J Epidemiol. 2003; 158:915-920. [PubMed: 14585769]

13. Rosenbaum PR, Rubin DB. The central role of the propensity score in observational studies for causal effects. Biometrika. 1983; 70:41-55.

14. Brookhart MA, Schneeweiss S, Rothman KJ, et al. Variable selection for propensity score models. Am J Epidemiol. 2006; 163:1149-1156. [PubMed: 16624967]

15. Parsons, L. Reducing Bias in a Propensity Score Matched-Pair Sample Using Greedy Matching Techniques. Twenty-Fifth Annual SAS Users' Group International Conference; Cary, NC: SAS Institute; 2000. p. 1166-1171.

16. Dormuth CR, Carney G, Carleton B, et al. Thiazolidinediones and fractures in men and women. Arch Intern Med. 2009; 169:1395-1402. [PubMed: 19667303]

17. Lipscombe LL, Gomes T, Levesque LE, et al. Thiazolidinediones and cardiovascular outcomes in older patients with diabetes. JAMA. 2007; 298:2634-2643. [PubMed: 18073359]

18. Solomon DH, Cadarette SM, Choudhry NK, et al. A cohort study of thiazolidinediones and fractures in older adults with diabetes. J Clin Endocrinol Metab. 2009; 94:2792-2798. [PubMed: 19470635]

19. Guess HA. Exposure-time-varying hazard function ratios in case-control studies of drug effects. Pharmacoepidemiol Drug Saf. 2006; 15:81-92. [PubMed: 16287211]

20. Robins JM, Hernan MA, Brumback B. Marginal structural models and causal inference in epidemiology. Epidemiology. 2000; 11:550-560. [PubMed: 10955408] 
21. Brookhart MA, Patrick AR, Dormuth C, et al. Adherence to lipid-lowering therapy and the use of preventive health services: an investigation of the healthy user effect. Am J Epidemiol. 2007; 166:348-354. [PubMed: 17504779]

22. Sturmer T, Rothman KJ, Glynn RJ. Insights into different results from different causal contrasts in the presence of effect-measure modification. Pharmacoepidemiol Drug Saf. 2006; 15:698-709. [PubMed: 16528796]

23. Aberra FN, Brensinger CM, Bilker WB, et al. Antibiotic use and the risk of flare of inflammatory bowel disease. Clin Gastroenterol Hepatol. 2005; 3:459-465. [PubMed: 15880315]

24. Lewis JD, Aberra FN, Lichtenstein GR, et al. Seasonal variation in flares of inflammatory bowel disease. Gastroenterology. 2004; 126:665-673. [PubMed: 14988820] 

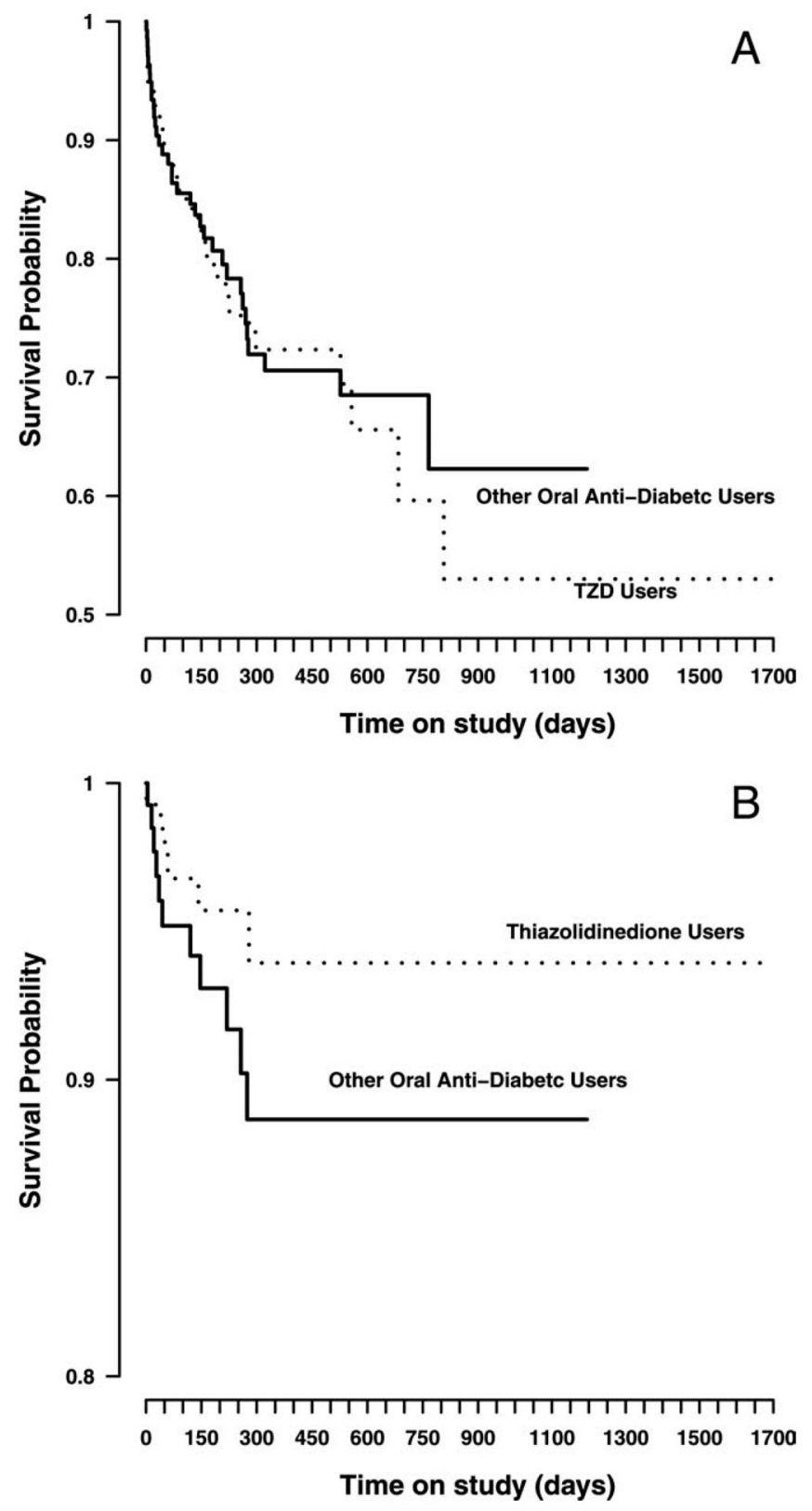

FIGURE 1.

Kaplan-Meier survival curves of individuals diagnosed with diabetes and UC comparing relapse-free survival for those treated with thiazolidinediones (hatched lines) compared to other oral antidiabetic medications. Hazard ratios were estimated using Cox proportional hazards regression after matching on propensity score. (A) Relapse is defined by the composite outcome of a new prescription for an oral or rectal aminosalicylates, oral steroids, or infliximab, or colectomy $(\mathrm{HR}=1.05,95 \% \mathrm{CI}$ : 0.66, 1.68). (B) Relapse is defined as a new prescription for oral steroids only (HR $=0.53,95 \% \mathrm{CI}: 0.20,1.44)$. 
TABLE 1

Characteristics of Prevalent Thiazolidinedione and Other Oral Anti-Diabetic Users with Ulcerative Colitis After Matching on Propensity Score

\begin{tabular}{|c|c|c|c|c|}
\hline & \multicolumn{2}{|c|}{ TZD users } & \multicolumn{2}{|c|}{ Other OAD users } \\
\hline & $N=\mathbf{1 3 8}$ & $\%$ & $N=138$ & $\%$ \\
\hline \multicolumn{5}{|l|}{ Demographic information } \\
\hline Age in years (mean $\left.\left(\mathrm{SE}^{*}\right)\right)$ & \multicolumn{2}{|c|}{$53.8(7.0)$} & \multicolumn{2}{|c|}{$54.0(7.2)$} \\
\hline Female gender & 60 & 43.5 & 69 & 50 \\
\hline Region - East & 33 & 23.9 & 33 & 23.9 \\
\hline Region - Midwest & 42 & 30.4 & 38 & 27.5 \\
\hline Region - South & 45 & 32.6 & 47 & 34.1 \\
\hline Region - West & 18 & 13.1 & 20 & 14.5 \\
\hline Medicaid & 1 & 0.7 & 2 & 1.5 \\
\hline \multicolumn{5}{|l|}{ Prior medication use ( 90 days before study entry) } \\
\hline Prior insulin use & 24 & 17.4 & 22 & 15.9 \\
\hline Prior immunomodulator use & 6 & 4.4 & 3 & 2.2 \\
\hline Prior methotrexate use & 0 & 0 & 1 & 0.7 \\
\hline Prior entocort use & 0 & 0 & 5 & $3.6^{*}$ \\
\hline Prior salicylate use & 87 & 63 & 84 & 60.9 \\
\hline Prior adalimumab use & 0 & 0 & 0 & 0 \\
\hline \multicolumn{5}{|l|}{ Prior comorbid conditions ( 180 days before study entry) } \\
\hline Peripheral vascular disease & 6 & 4.4 & 5 & 3.6 \\
\hline Cardiovascular disease & 5 & 3.6 & 5 & 3.6 \\
\hline Myocardial infarction & 3 & 2.2 & 4 & 2.9 \\
\hline Congestive heart failure & 5 & 3.6 & 3 & 2.2 \\
\hline Dementia & 0 & 0 & 0 & 0 \\
\hline Chronic obstructive pulmonary disease & 12 & 8.7 & 6 & 4.4 \\
\hline Rheumatologic disease & 0 & 0 & 1 & 0.7 \\
\hline Peptic ulcer disease & 1 & 0.7 & 2 & 1.5 \\
\hline Diabetes (mild to moderate) ${ }^{a}$ & 121 & 87.7 & 105 & 76.1 \\
\hline Diabetes with chronic complications ${ }^{a}$ & 13 & 9.4 & 13 & 9.4 \\
\hline Retinopathy, nephropathy, or neuropathy & 8 & 5.8 & 8 & 5.8 \\
\hline Hemiplegia or paraplegia & 0 & 0 & 0 & 0 \\
\hline Mild liver disease & 0 & 0 & 2 & 1.5 \\
\hline Moderate or severe liver disease & 28 & 20.3 & 22 & 15.9 \\
\hline Renal disease & 4 & 2.9 & 4 & 2.9 \\
\hline Any malignancy, including lymphoma and leukemia & 5 & 3.6 & 6 & 4.4 \\
\hline Metastatic solid tumor & 0 & 0 & 1 & 0.7 \\
\hline AIDS & 0 & 0 & 0 & 0 \\
\hline \multicolumn{5}{|l|}{ Calendar time } \\
\hline Year 2000 & 2 & 1.5 & 1 & 0.7 \\
\hline Year 2001 & 4 & 2.9 & 5 & 3.6 \\
\hline
\end{tabular}




\begin{tabular}{|c|c|c|c|c|}
\hline & \multicolumn{2}{|c|}{ TZD users } & \multicolumn{2}{|c|}{ Other OAD users } \\
\hline & $N=138$ & $\%$ & $N=\mathbf{1 3 8}$ & $\%$ \\
\hline Year 2002 & 18 & 13 & 18 & 13 \\
\hline Year 2003 & 50 & 36.2 & 59 & 42.8 \\
\hline Year 2004 & 56 & 40.6 & 46 & 33.3 \\
\hline Year 2005 & 8 & 5.8 & 9 & 6.5 \\
\hline \multicolumn{5}{|l|}{$\underline{\text { Healthcare utilization }}$} \\
\hline Days-in-claims (0-5) & 36 & 26.1 & 38 & 27.5 \\
\hline Days-in-claims (6-11) & 59 & 42.8 & 61 & 44.2 \\
\hline Days-in-claims (>12) & 43 & 31.2 & 39 & 28.3 \\
\hline Total prescriptions $(0-13)$ & 44 & 31.9 & 40 & 29 \\
\hline Total prescriptions (14-27) & 44 & 31.9 & 48 & 34.8 \\
\hline Total prescriptions (>28) & 50 & 36.2 & 50 & 36.2 \\
\hline
\end{tabular}

TZD, thiazolidinedione; OAD, other oral antidiabetic; SD, standard deviation.

${ }^{*} P<0.05$.

${ }^{a}$ Categories are not mutually exclusive based on differences in cohort definitions and the Deyo implementation of the Charlson comorbidity index. 


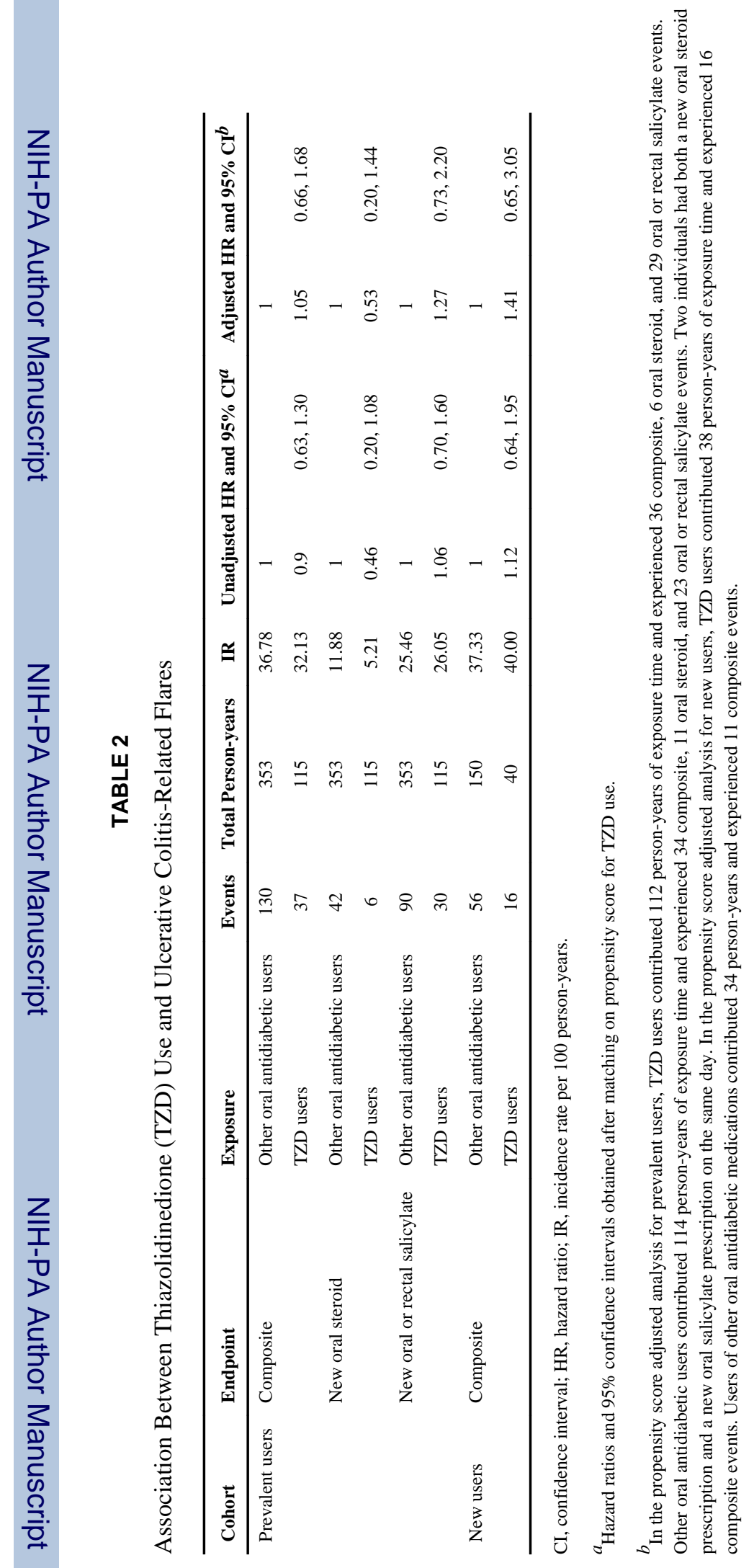

Inflamm Bowel Dis. Author manuscript; available in PMC 2012 March 1. 